\title{
ACADEMIC PERFORMANCE PREDICTION APPLICATION (APPA)
}

\author{
Dr. Tejashree T. Moharekar \\ Assistant Professor \\ Department of Mathematics \\ Shivaji University, Kolhapur \\ tejashreesawant2@gmail.com
}

\author{
Dr. Urmila R. Pol \\ Assistant Professor \\ Department of Co mputer Science \\ Shivaji University, Kolhapur \\ urmilec@gmail.com
}

\begin{abstract}
The research study offers a thorough description of the process of deployment after training and testing of the classification model respectively. The performance of students is a crucial prerequisite to help students that don't perform well in the examination and can impact the final semester result. To overcome the difficulties they come across while learning and assist them to achieve the best results. The researcher uses the advantages of the React-Native platform to build an "APPA" mobile application capable of delivering student performance prediction-related solutions. It also provides a proposed model of student academic success prediction. The further study highlights the further scope of the mobile App built for predicting student performance.
\end{abstract}

KEYWORDS: Academic, performance, prediction, application, APPA, machine learning, Mobile Application Development Life Cycle (MDLC)

\section{INTRODUCTION}

System implementation is one of the most challenging phases for machine learning to achieve value. It is necessary to quickly deploy model into production in order to get the most value out of the machine learning model, so that an enterprise can start using them to make practical decisions. The deployment of established classification model is the process of making model accessible in production environments, where in our case it is mobile application they can provide predictions to other software systems. "No machine learning model is valuable, unless it 's deployed to production." - Luigi Patruno. Classification models can only produce university interest when the students obtain inputs from those models. Students are the end users in our research study as the developed model for predicting their last semester post-graduation outcome 
which is very important from a career perspective. During the analysis, it was observed that the model for university faculties is being developed to forecast student output based on the past outcome. But this research has developed a student machine learning model that can predict outcomes before the final semester exam and work accordingly to improve the final semester outcome. And the data mining model can be said to be effective if and only if it is implemented and the students are provided with its predictions.

\section{METHODOLOGY}

Researcher has used different classification methods to carry out a classification prediction model on student dataset, plotting results and then calculate the model performance on the test data in python environment using different evaluation metrics. Then developed model implementation and application is build. The conceptual model offered for APPA is as follows.

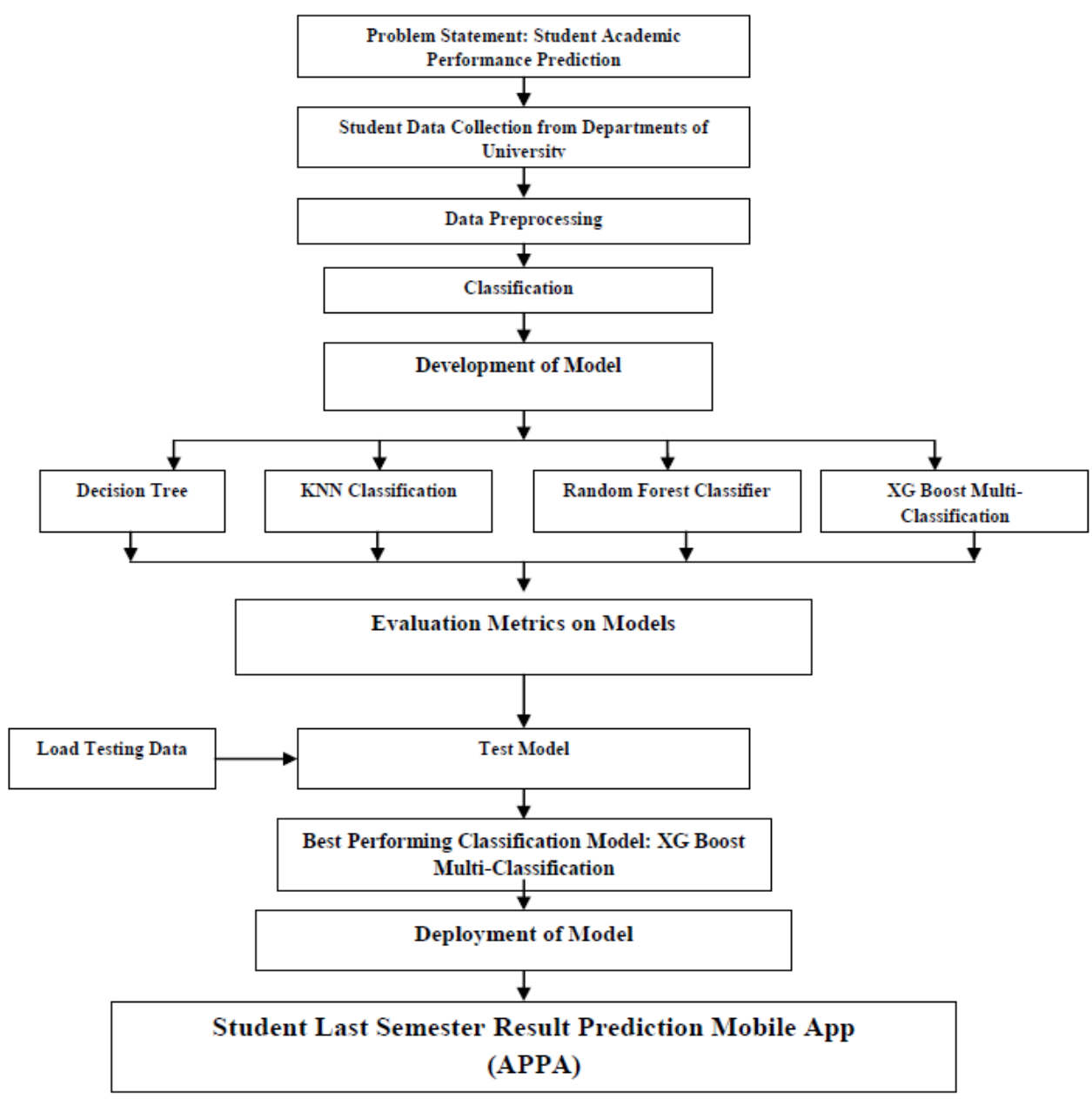

Figure 1. Conceptual Model For APPA (Source: (Sawant \& Pol, 2021) 
Researcher have found in this study that XGBoost Multi-Classification offers the best accuracy when trained on a dataset of 20 key components compared to Random Forest Classification, kNN Classification and Decision Tree Classification. Researcher has therefore implemented XGBoost Classification which is the best performing model seen in the model evaluation phase into an mobile app for students to predict their results on their smart mobile devices. Using this, researchers can approximately achieve an accuracy of 86 per cent. Now to use this model with new unknown data we need to save the model so that the values can be estimated by the researcher later (Sawant \& Pol, 2021).

COMPARATIVE RESULTS FOR DIFFERENT CLASSIFICATION MODEL

\begin{tabular}{|c|c|c|c|c|c|c|c|}
\hline & & \multicolumn{2}{|c|}{ On Training Data } & \multicolumn{3}{c|}{ On Testing Data } \\
\hline Algorithms & Feature Set & Accuracy & Precision & Recall & Accuracy & Precision & Recall \\
\hline DT Classifier & Label-I & $78.24 \%$ & $52.25 \%$ & $48.37 \%$ & $77.14 \%$ & $51.56 \%$ & $47.25 \%$ \\
\hline KNN Classifier & Label-I & $62.16 \%$ & $47.28 \%$ & $46.31 \%$ & $61.82 \%$ & $46.12 \%$ & $45.22 \%$ \\
\hline RF Classifier & Label-I & $60.09 \%$ & $31.80 \%$ & $23.45 \%$ & $59.68 \%$ & $30.41 \%$ & $19.05 \%$ \\
\hline XGBT Classifier & Label-I & $75.25 \%$ & $60.74 \%$ & $60.32 \%$ & $75.15 \%$ & $59.72 \%$ & $59.42 \%$ \\
\hline
\end{tabular}

Table 1: Results for different Classification model

Source: (Sawant \& Pol, 2021)

Researcher has deployed model using the open-source Django platform that is accessible in python language. Researchers also developed a mobile app that consumes this Django-based REST API and provides visitors with the prediction. For this purpose, the researchers have selected the architecture for deploying the basic app. 


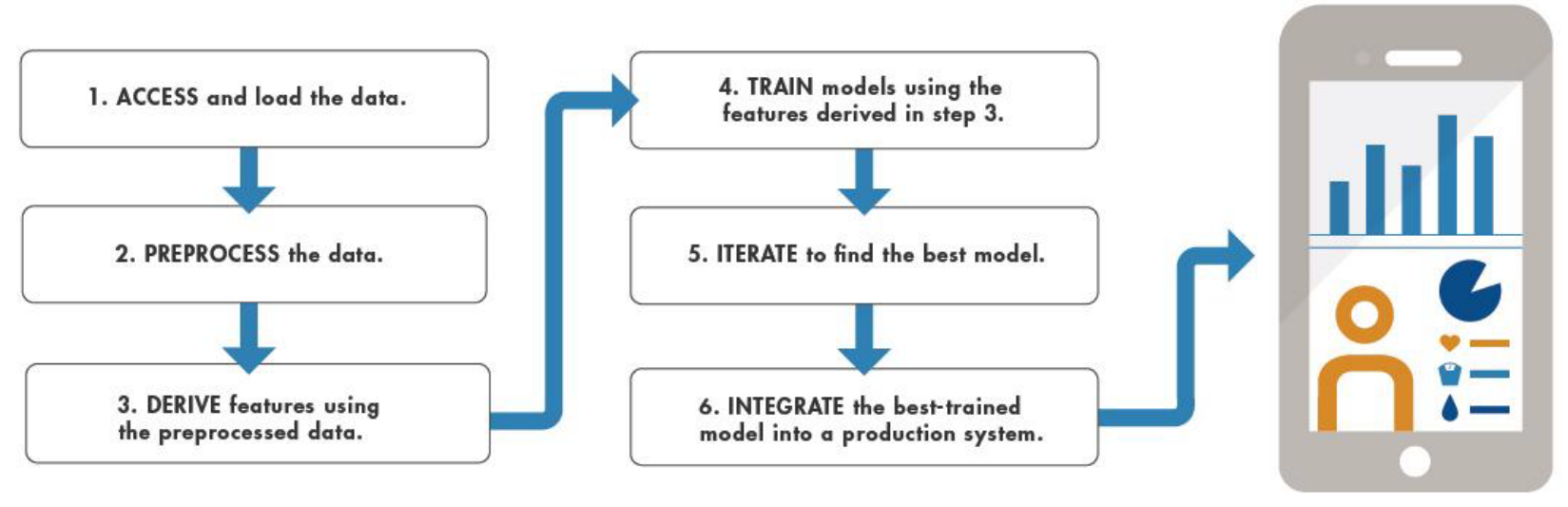

Figure 2. Model Deployment Process

\section{CLASSIFICATION MODEL IN PRODUCTION}

Predictive models are intended to drive real business value by constantly supporting predictions in production environments, or producing predictions on small problem immediately. Researchers have put into production our selected model which means deploying the classification model and making the model available to end users or systems. Using a supervised learning algorithm and the popular scikit-learn Python library, the researcher uses the labeled input data to train a classification prediction model to incorporate the model into a simple reaction native application, which is then deployed to a cloud production environment using Heroku open source platform as service. The following figure shows the process at a high level.

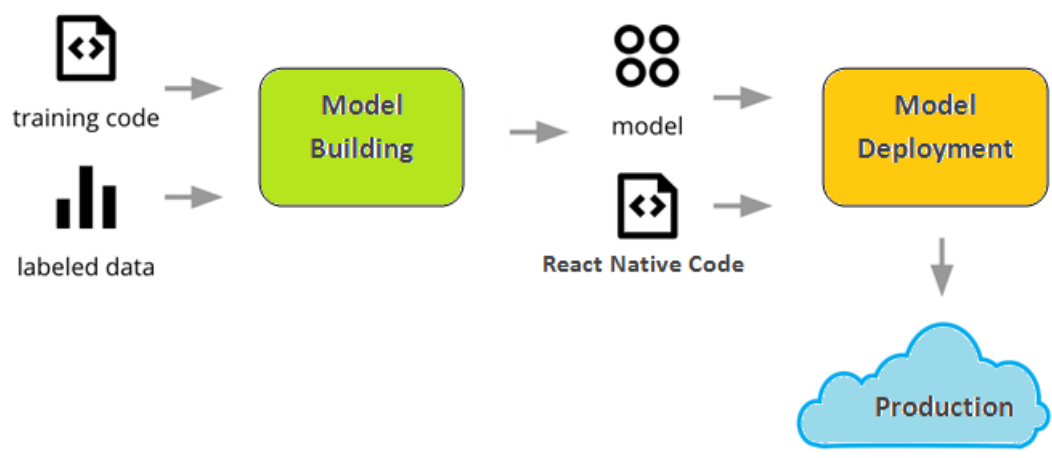

Figure 3. The High-level Process

Work in this emphasizes the research methodology planning process (CRISP-DM) followed to achieve the aims of this study. After exploratory of the data and framing the research objective, 
DM techniques are applied to the student data set in the previous chapter. And eventually best performing algorithm is chosen for the further step of deployment. This research study was done primarily by researching and using the React JS framework and React environment to develop the React Native App, which is one of Frontends' best frameworks worldwide. The application focuses on using React-Native to construct the single-page application for academic performance prediction which is a requirement of a modern mobile application.

\section{SERIALIZING CLASSIFICATION MODEL}

The pickle method constructs binary functions which mounts and unmounts a Python object. "Pickling" is the method of transforming a Python object structure into a byte stream, and "unpickling" is the reverse procedure by which a byte stream (from a binary file or byte-like object) is transformed back into an object hierarchy. Pickling (and unpickling) is referred to as "serialization," but the words used here are "pickling" and "unpickling" to prevent ambiguity. Serialization is a method of transforming python objects into a storage format that allows recovery of the python object's original structure from the stored format. Researchers need to serialize trained model to be able to use it from our API. Researcher saved a qualified model of the classifier to a disk using pickle. It can then be later reloaded, and used as if we had trained it.

\section{DEVELOPMENT OF MOBILE APPLICATION INTERFACE}

Developing app also involves developing one for Android, and one for iOS, the two different mobile device having operating systems. The two applications may have the same layout and logic, but several user interface (UI) components will differ, and the applications need to be developed in two different languages themselves. This process is gruesome as creating two applications is time consuming, and requires two different sets of knowledge. Researcher has developed an app with the newly released React Native framework, which can create both the iOS and Android app by compiling the code written in React. It is uncomplicated to develop and you can create an application in a very short time, and compile it for both Android and iOS. Researcher has used React, Django, and the Django REST Framework to build a modern mobile application with a separate REST API backend and frontend. A POST request is made at a specified link when the form is submitted, and we get the predicted output. Finally, the endpoint functions were written by researcher. When a user sends the form by entering all attributes the 
predict function will be called and the predict value will result in a JSON format output. Researcher used the same values to display the user's performance on user interface as the result of the final semester shown in figure. The main page in this phase contains a form for students to enter their basic details, such as personal attributes, academic, social attributes and institutional/ school / university attributes etc. Under the tabs Personal Attributes, Academic, Social Attributes and Institutional features, number of responses were collected to predict the results based on those factors respectively.
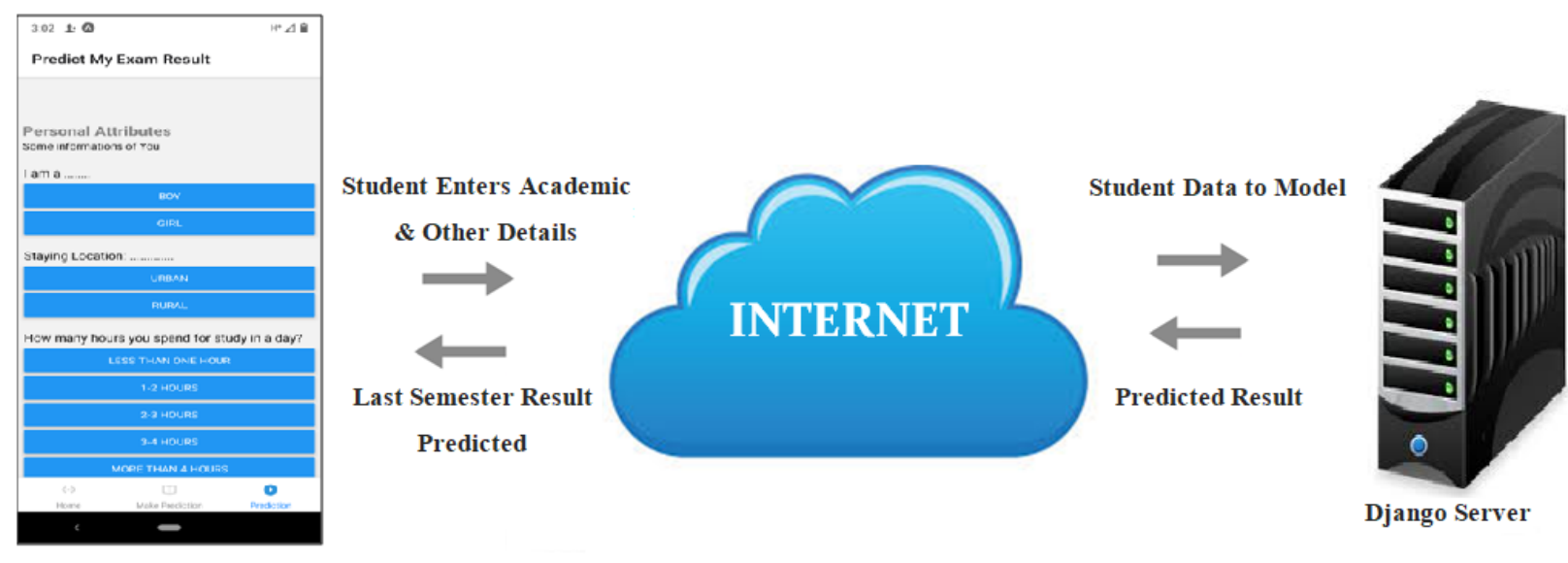

Figure 4: Mobile Application Interface

As soon as the frontend receives the data and student presses the submit button developed classification model gets into collection of testing as the researcher used API to call this service. This service can be called a prediction service, as it predicts the student's results after all the data it requires to predict is successfully received.

\section{MOBILE APPLICATION DEVELOPMENT LIFE CYCLE}

Mobile applications to deploy in smart phones are being developed. Given the growing need for mobile applications and the as sociated development complexity, a specialized mobile application development life cycle framework is extremely important. Mostly used software development models are waterfall model, iterative model, prototyping model and spiral model etc. A distinct lifecycle model needs to be developed for mobile applications: development of mobile application incorporates a variety of functionalities (Sommerville, 2016). Slalom Consulting introduced the mobile application development lifecycle describing as "Enterprise Mobile 
Application Lifecycle": the phase from beginning to last in development of mobile application which involves discovery, design, creation / testing and delivery of four phases (KARTHIK_P, 2016). Mobile Device Development Lifecycle (MADLC) has applied in the current research to develop a formalized lifecycle protocol that will enable developers of mobile applications to create high-end applications. Different procedure steps are shown in figure. Phases were addressed in MADLC which consists of various tasks and activities that take place in the next section during the process.

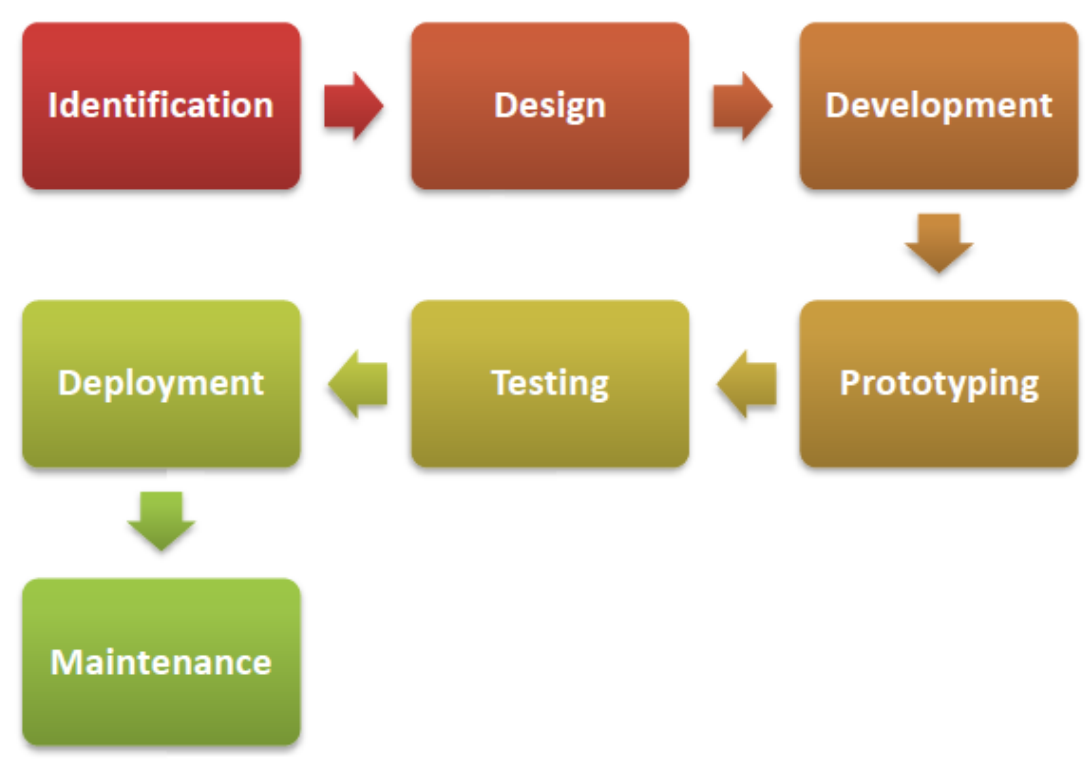

Figure5. Mobile Application Development Life Cycle (MADLC) (Vithani \& Kumar, 2014)

\section{ACADEMIC PEFORMANCE PREDICTION APPLICATION (APPA) MADLC}

It is the phases in mobile applications development with proper way which integrates many functionalities better than the desktop development applications, (Fenty, 2014) (Muhammad Adil, 2015) (Nurfarah Ain, 2015) projected to facilitate an efficient way in building application for mobile application development. Researcher will follow MADLC for the developed Academic Performance Prediction Application (APPA). MADLC is classified into the following seven units to process step by step starting with identification phase to maintenance phase. MADLC is applied successfully in creating Android apps for over a year. The preliminary indicators are that the mobile developers would benefit the development cycle with effective and stable applications (Muhammad Adil, 2015). 


\section{IDENTIFICATION PHASE}

Identification phase helps to put forward a new concept or changes to the current application (Vithani \& Kumar, 2014). The role and responsibilities to do analysis and categorization of application concept and ideas are performed by the developer (Fenty, 2014). In this study, the program focuses primarily on predicting last-semester outcome for students based on the classification model being educated. A solution to this study's underlying problem to predict academic student results, the researcher uses the advantages of the React-Native platform to build a "APPA" mobile application capable of delivering student performance prediction solution. The created app was built for Android and iOS, and the results produced represent satisfactory user experience in both platforms. The methodology proposed was implemented with the help of React-native.

\section{DESIGN PHASE}

The idea behind this phase is to design the application for further development (Vithani \& Kumar, 2014). Once the identification phase is done the next phase is design which includes the blueprint of the whole application. The flow of the data across the system is shown neatly. In this phase the concept is mapped to follow it as per the architecture. It is also most essential phase of the application development. (Muhammad Adil, 2015)The practicability of application on all available mobile platforms is identified. In this phase, researcher needs to propose the required database system. Also the design for the graphical user interface is determined in this phase. The figure shows the data flow diagram in the developed application. Student will enter as a frontend screen form all the options provided by the researcher, then he / she should click on the SUBMIT button within a second student will see the predicted result for the upcoming final semester as shown in the last screen. Student will make proper decisions and take appropriate actions to enhance quality learning with result display and thus improve student performance. Faculty should also inspire students to do better throughout the semester and to direct them accordingly. 


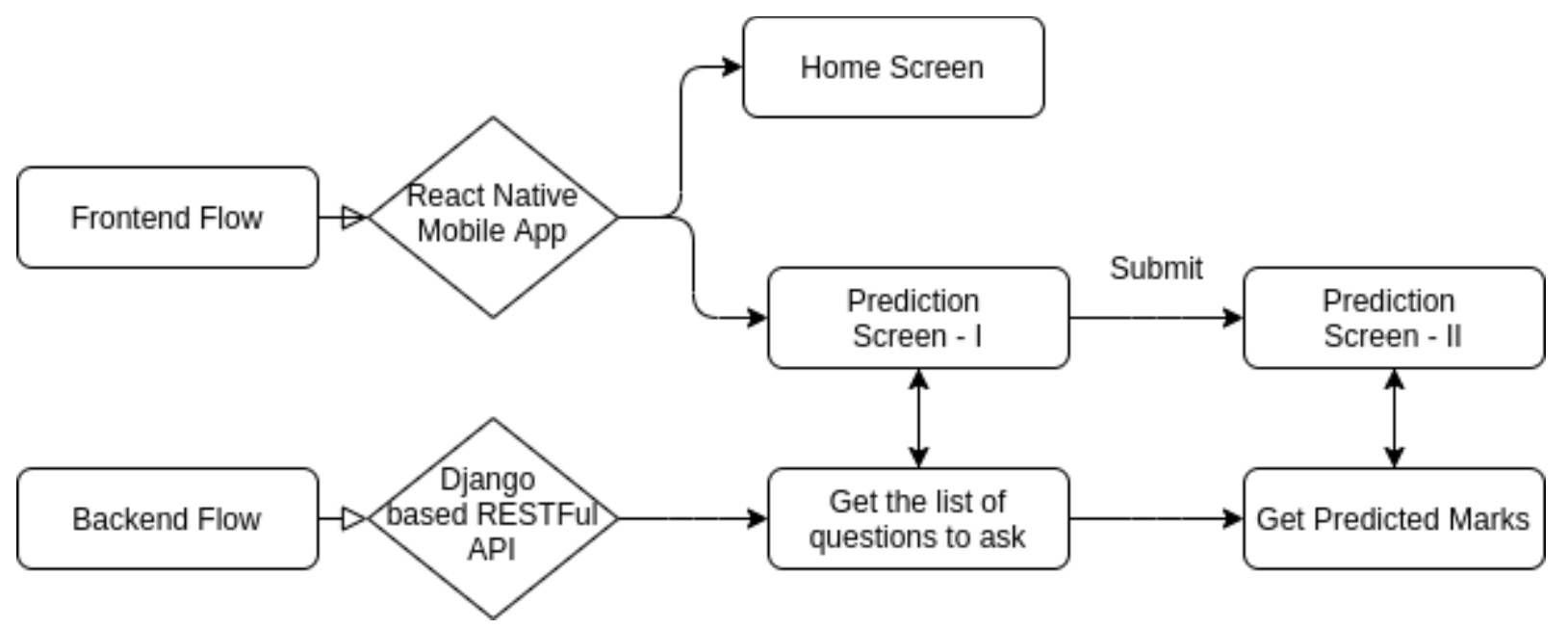

Figure 6. Flowchart of APPA

\section{DEVELOPMENT PHASE}

The application's coded in this process. Simultaneously coding for different modules can be done (Nurfarah Ain, 2015). There is a resource that has been used in the program development to support the person involved in project creation. Researcher did prediction models on Anaconda Framework in the Jupyter notebook. The open source technology needed is the React Native framework and ATOM editor for the building the mobile app. ANACONDA Enterprise is an industry-ready, efficient, and scalable advanced analytic platform that enables organizations to identify, coordinate, and execute business analytics for decision making. It is a platformagnostic, so whether you're on Windows, MacOS or Linux, you can use this. Build ML / AI pipelines in a centralized production environment which is scalable from the laptops to multiple nodes. The Jupyter Notebook: Jupyter Notebook work as a web application an open source that allows the development and sharing of live data, calculations, graphical representation. It helps in data pre-processing, exploratory data analysis, data modeling, machine learning and data visualization etc. It is an incredibly potent tool for interactive data mining development and presentation. The Jupyter project is the successor to the previous IPython Notebook which was first published in 2010 as a prototype. Within Jupyter Notebooks you can use many different programming languages. Python: Researcher has selected python for model development because python is versatile with applications, simple scripts and links to datasets from the top programming languages of 2020. In Python, you can create pretty much anything. Plus it's also 
used to create artificial intelligence neural networks. Part of this is due to the large digital library, but also because it is compatible with most major databases and systems. Last but not least, it is a language with very simple syntax, which makes it easy to read. No wonder it is at the top of the list. Python has steadily risen through the ranks of the programming languages over the last few years. Each other language has meanwhile remained stable, with a gradual rise across the board. The market for programmers is apparently always growing. The market for creation of mobile apps is growing tremendously and both academic researchers and companies are creating new technologies and programming tools that are open source and have simple user interface. Of the various android platforms, iOS and windows are the most common and commonly used. Developing an application requires several types of behavior like choosing the correct technology (programming language) and selecting a specific platform. Mobile application development is close to a conventional desktop because of the available software and hardware limitations, with certain difficulties and complexities involved. This list of software has used to propose the system interface, and to program the module. The application's graphical user interface is presented taking into account different controls in mind. The application integrated with different functionalities of designing the UI and application icons as system inputs.

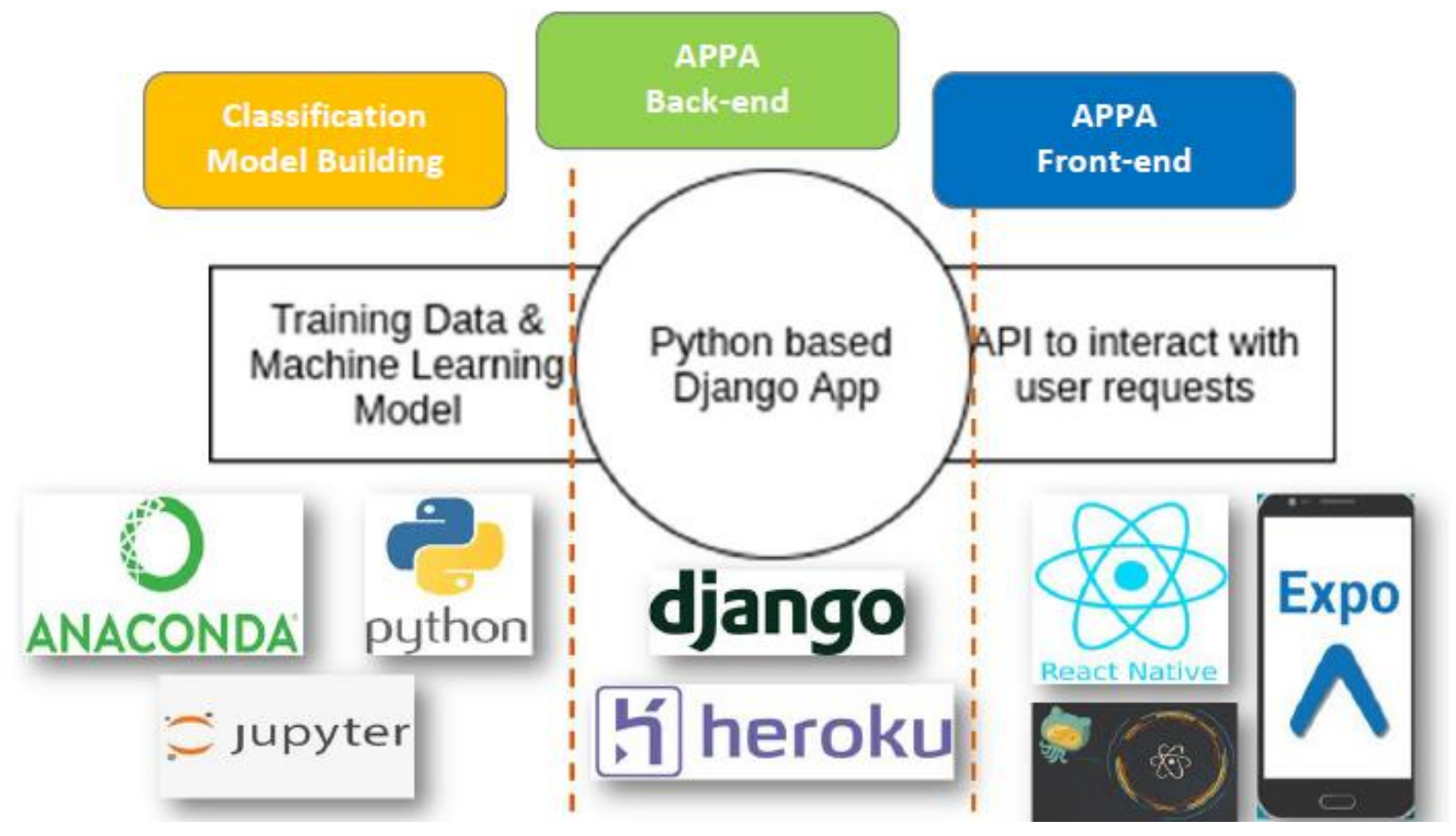

Figure 7. Technology Full Stack for APPA 
Researchers have created an Android app MVP that helps students prepare well for their semester wise test. A student will have to submit their information like personal details and publish his / her expected marks by the submission. This can help a student plan and upgrade to the academics in advance. Students may find it very helpful to gain insight into results using their Smartphone, as the number of mobile users is much greater than users of web browsers. The program offers a user friendly GUI.

Developed Application main page including different options present is shown below:

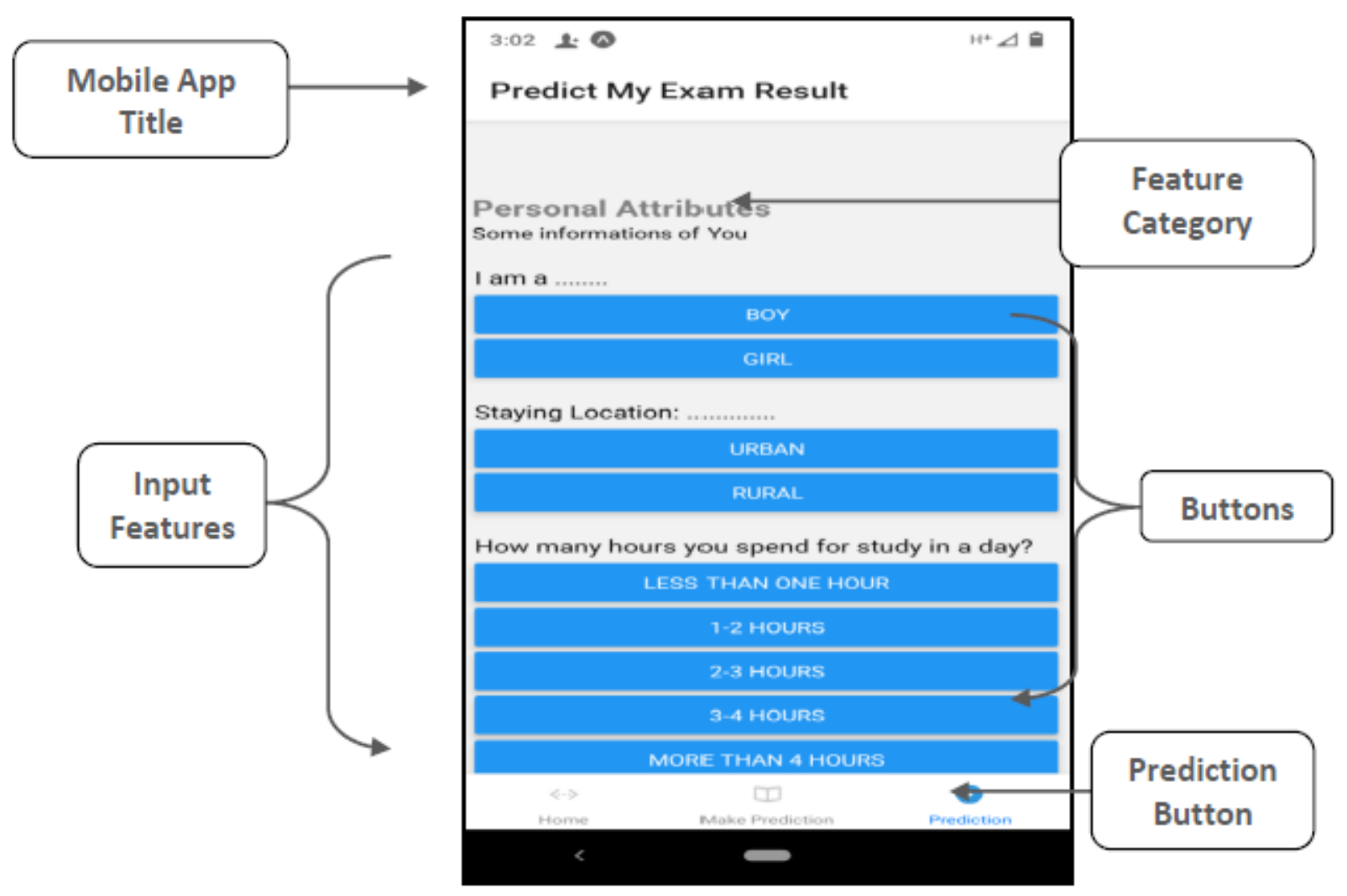

Figure 8. Functionality of Screen

\section{TESTING PHASE}

Testing been the most essential phases of any lifecycle for development (Vithani \& Kumar, 2014)(Muhammad Adil, 2015). The testing of the system or application is based on the blackbox approach which performs by using diagram called use-case diagram. (Fenty, 2014)Black- 
box testing approach is a basic feature of testing framework without having to look at the internal program frameworks. Such technique is used to estimate if the programming works correctly. Black-box computation process is a technique of developing data audits based on the criteria of application. The test data developed and implemented on the application, and the output is then verified to see whether the intended outcome is satisfactory.

\section{DEPLOYMENT PHASE}

The next stage of production is implementation. The program is ready for deployment, after completing the test and receiving the reviews. The developed application will be submitted to the respective application store for student adoption. One of the simplest and friendly direction is to achieve deployment is to create a REST API for the model, so that you can use the model from any platform that can interact with REST APIs. REST APIs is compatible for cross-platform integration meaning is that a many applications, such as mobile apps, web browsers, sales-force apps, etc., may use your model. There are a range of frameworks available for building REST APIs. Researcher takes advantage of the Django Framework and the Django REST API system. The reason for choosing Django is that both the core Django framework and the REST API framework are constantly being updated, have strong documentation and community, and are expected to continue to be popular.

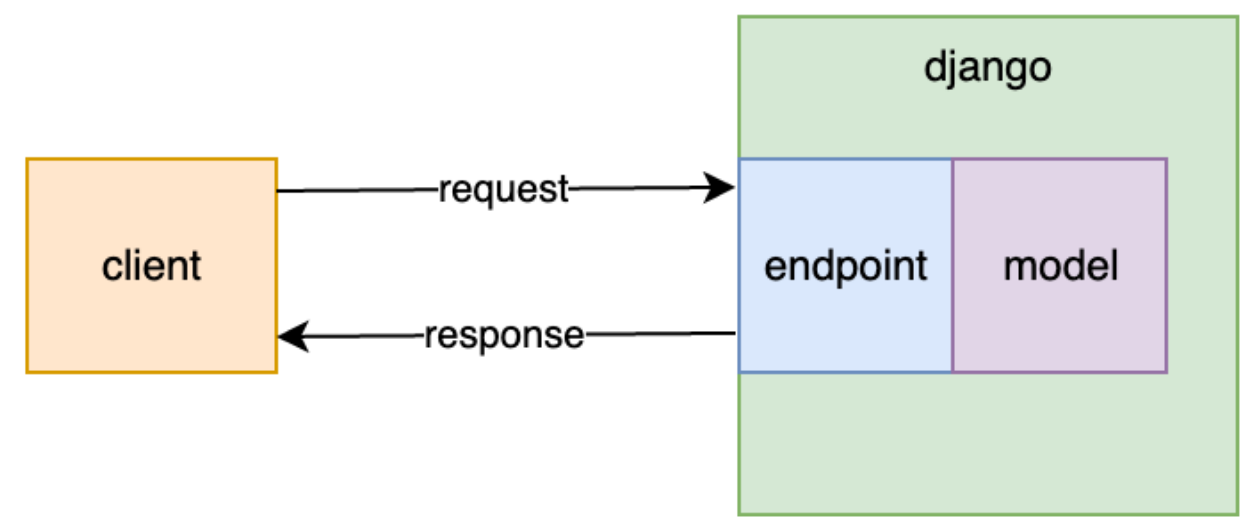

Figure 9. Django Architecture 
The architecture pattern for the Model-View - Controller program is widely used in Django because it is an efficient way to build a dynamic website (i.e. using stored data). MVC Modelmanages the dynamic data structure (i.e. database) View is what the user is able to see and interact with Controller is the middle man who takes inputs and transforms it to model or interface commands. Heroku is a server system works over cloud platform and offering built-in different data services and makes it a very powerful. Deployment and execution of various applications can be done using Heroku (Salesforce. 2016). It is use to execute and test deployed sites. "It lets us focus on our app \& deliver more significance to customers"-AdronPeckham. Git helps Heroku to interact straight with private local virtual environments for the complete uploading of applications to the cloud easy and fast.
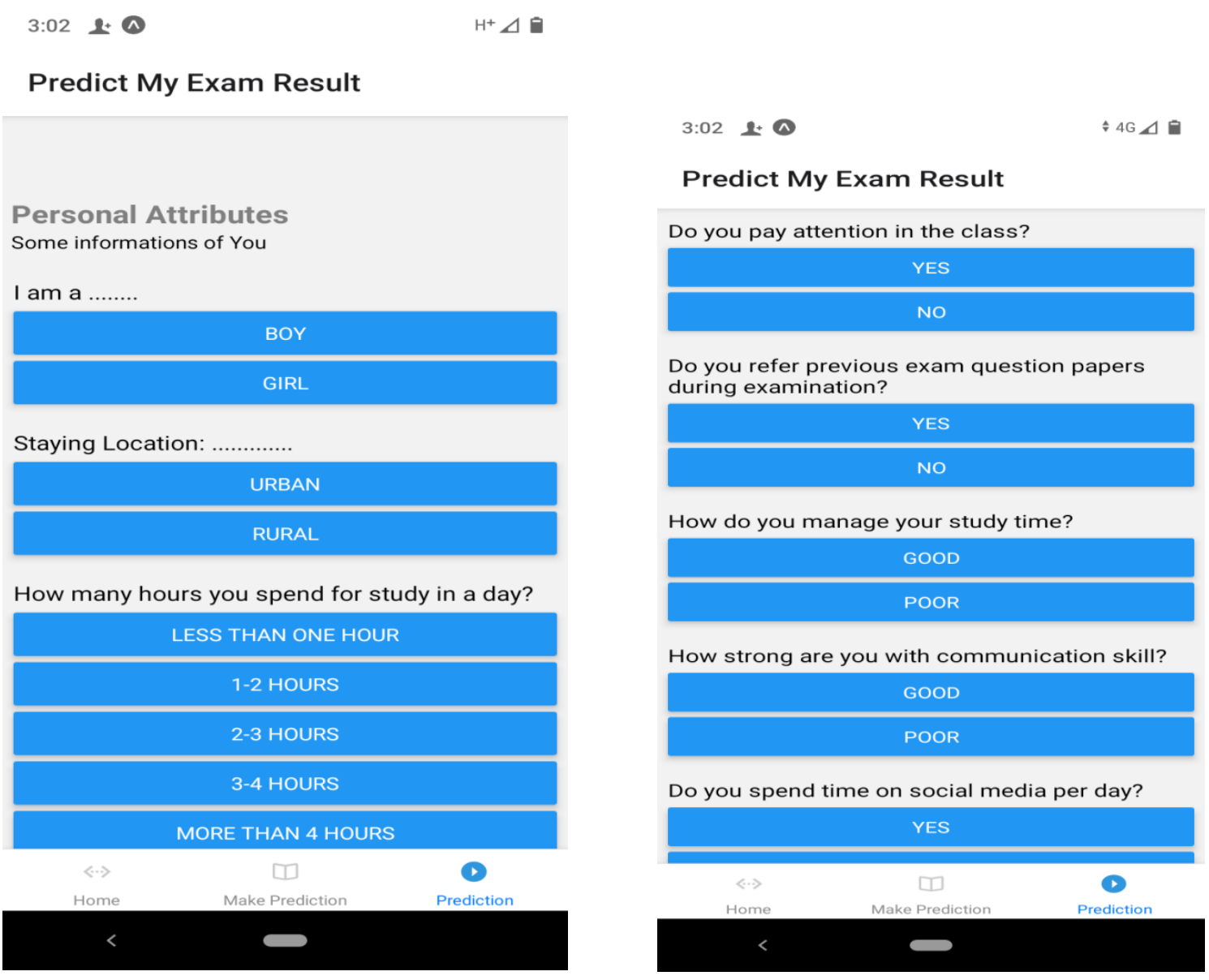
3:02 2. 1

$4 \mathrm{G} \Delta \mathrm{\theta}$

\section{Predict My Exam Result}

Do you spend time on social media per day?

YES

Do you take any online course material for study during exam?

YES

Do you suffer from health issues?

YES
SOMETIMES
NO

Do you feel stressful during exam?

YES

\section{Academic Attributes (Numericals}

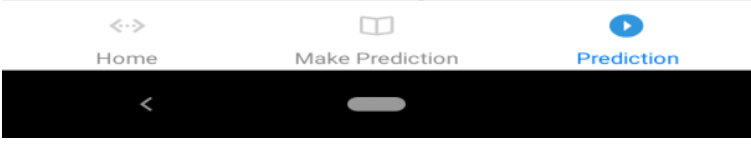

3:03 2 1

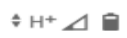

\section{Predict My Exam Result}

Academic Attributes

Some about past records

Have you joined any extra tuition to study?

YES
NO

How much is your attendance in class?

\begin{tabular}{c} 
ABOVE 70 \\
\hline BETWEEN 40-70 \\
ABOVE 40 \\
\hline
\end{tabular}

Do you take out your own notes for study in examination?

\begin{tabular}{|c|}
\hline YES, ALL THE TIME \\
\hline ONLY SOMETIMES \\
\hline NOT AT ALL \\
\hline
\end{tabular}

Social Attributes

What does your social circle say about you?

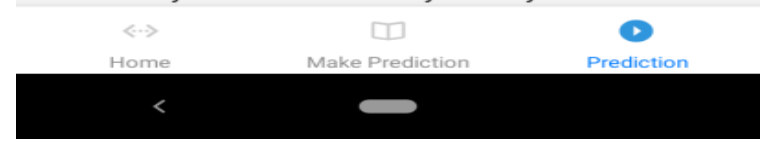

3:03 $1+1$

$4 \mathrm{G} \Delta$

\section{Predict My Exam Result}

\section{Academic Attributes (Numericals Input) \\ Some about past records \\ HSC marks (in \%) \\ 50.0 \\ SSC marks (in \%) \\ 50.0 \\ UG aggregate marks (in \%) \\ 75.0 \\ Post Graduate Semester I marks (in \%) \\ 60.0 \\ Post Graduate Semester II marks (in \%) \\ 70.0 \\ Post Graduate Semester III marks (in \%) 80.0}

\section{Academic Attributes}

Some about nast records

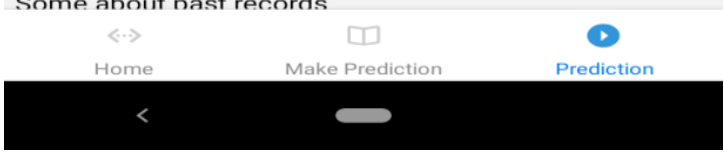

3:03 2.

\section{Predict My Exam Result}

Social Attributes

What does your social circle say about you?

Do you take family responsibility while learning?

\begin{tabular}{|c|} 
YES \\
\hline SOMETIMES \\
\hline NO \\
\hline
\end{tabular}

How is your financial status?

\begin{tabular}{|c|}
\hline LOW \\
\hline AVERAGE \\
HIGH \\
\hline
\end{tabular}

What is your family literacy status?

\begin{tabular}{l} 
LITERATE \\
ILLITERATE \\
\hline
\end{tabular}

Does your family member encourage you to perform better in examination?

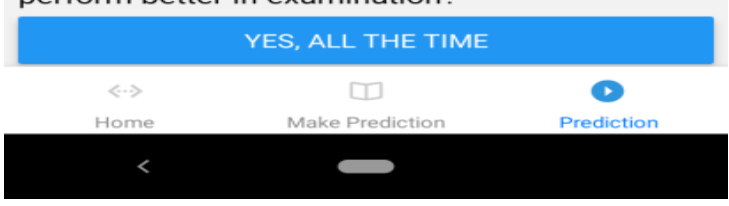




\section{3:03 2. 0 \\ $\mathrm{H}^{+} \Delta \mathrm{B}$}

\section{Predict My Exam Result}

Does your family member encourage you to perform better in examination?

\begin{tabular}{|c|}
\hline YES, ALL THE TIME \\
\hline ONLY SOMETIMES \\
NOT AT ALL \\
\hline
\end{tabular}

Does your friend encourage you to perform better in examination?

\begin{tabular}{|c|}
\hline YES, ALL THE TIME \\
\hline ONLY SOMETIMES \\
NOT AT ALL \\
\hline
\end{tabular}

Do you study by discussing with friends during exam?

\begin{tabular}{|c|} 
YES \\
\hline SOMETIMES \\
\hline NOT AT ALL
\end{tabular}

\section{Institute/School Attributes}

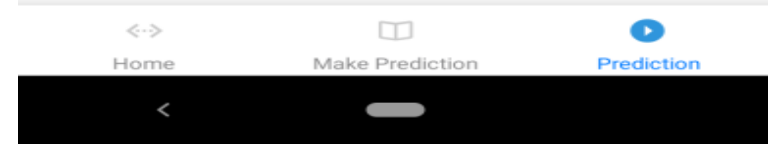
3:03 2.
$\mathrm{H} \boldsymbol{\theta}$

\section{Predict My Exam Result}

Do you understand/follow method of teaching used in class?

\begin{tabular}{|c|}
\hline UNDERSTANDABLE \\
\hline NOT UNDERSTANDABLE \\
NEUTRAL \\
\hline
\end{tabular}

Exam result gets affected due to absenteeism (teaching-learning gap)?

\begin{tabular}{|c|}
\hline AGREE \\
\hline NOT SURE \\
\hline DISAGREE \\
\hline
\end{tabular}

How do you feel in Exam hall during examination?

\begin{tabular}{|c|}
\hline VERY MUCH STRESSFUL \\
\hline NEUTRAL \\
RELAXED
\end{tabular}

Does your teacher encourage you to perform better in examination?

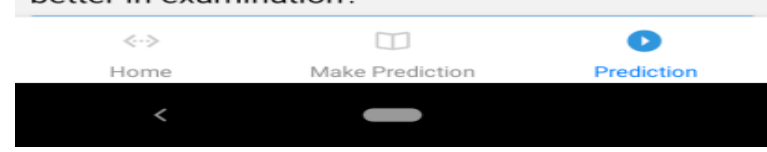

$$
\text { 3:03 2. C }
$$$$
\mathrm{H}^{+} \Delta \mathbf{E}
$$

\section{Predict My Exam Result}

\section{Institute/School Attributes}

Finally, tell us about your Institution?

How long it takes to reach to university?

\begin{tabular}{|c|}
\hline LESS THAN 1 HOUR \\
\hline $1-2$ HOURS \\
\hline $2-3$ HOURS \\
\hline 3-4 HOURS \\
\hline MORE THAN 4 HOURS \\
\hline
\end{tabular}

Mention your medium of Instruction in School Education

\begin{tabular}{l} 
ENGLISH LANGUAGE \\
\hline MARATHI LANGUAGE
\end{tabular}

Do you understand/follow method of teaching used in class?

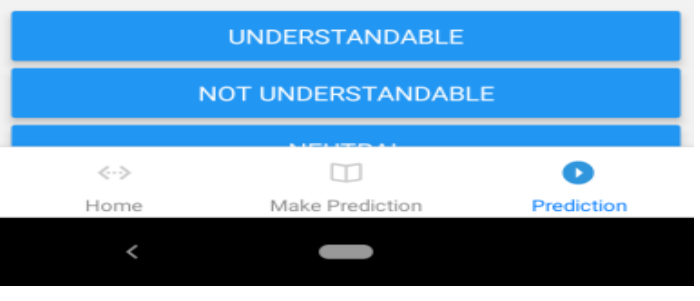

3:03 2.

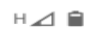

\section{Predict My Exam Result}

Does your teacher encourage you to perform better in examination?

\begin{tabular}{|c|}
\hline YES, ALL THE TIME \\
\hline ONLY SOMETIMES \\
NOT AT ALL \\
\hline
\end{tabular}

Does any expert motivate you to perform better in examination?

\begin{tabular}{l} 
YES, ALL THE TIME \\
\hline ONLY SOMETIMES \\
NOT AT ALL
\end{tabular}

How you think is your Course Syllabus?

\begin{tabular}{|c|}
\hline LESS APPROPRIATE \\
\hline AVERAGE \\
\hline MORE APPROPRIATE \\
\hline
\end{tabular}

Does Cultural Festival Holidays impacts on examination?

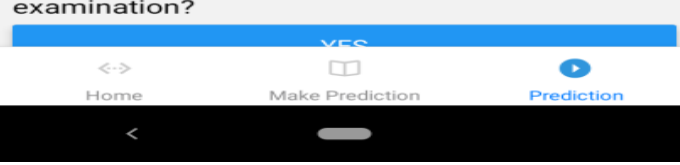


3:04 2. 0

Predict My Exam Result

\begin{tabular}{|c|}
\hline ONLY SOMETIMES \\
NOT AT ALL
\end{tabular}

How you think is your Course Syllabus?

\begin{tabular}{c} 
LESS APPROPRIATE \\
AVERAGE \\
\hline MORE APPROPRIATE
\end{tabular}

Does Cultural Festival Holidays impacts on examination?

YES
NO

Will you like incentive programs while learning?

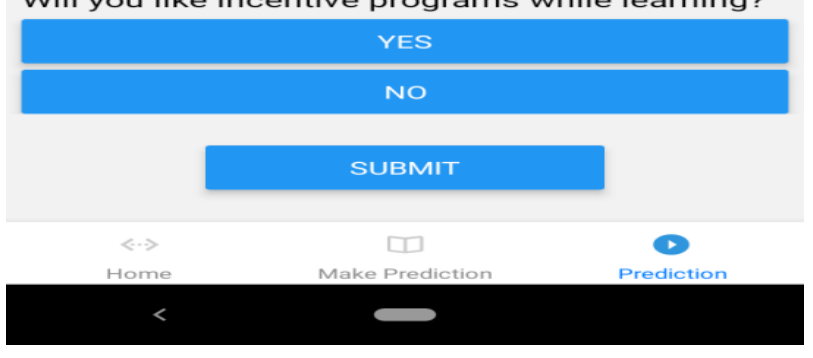

$7: 18 \rightleftharpoons 60$

Predict My Exam Result

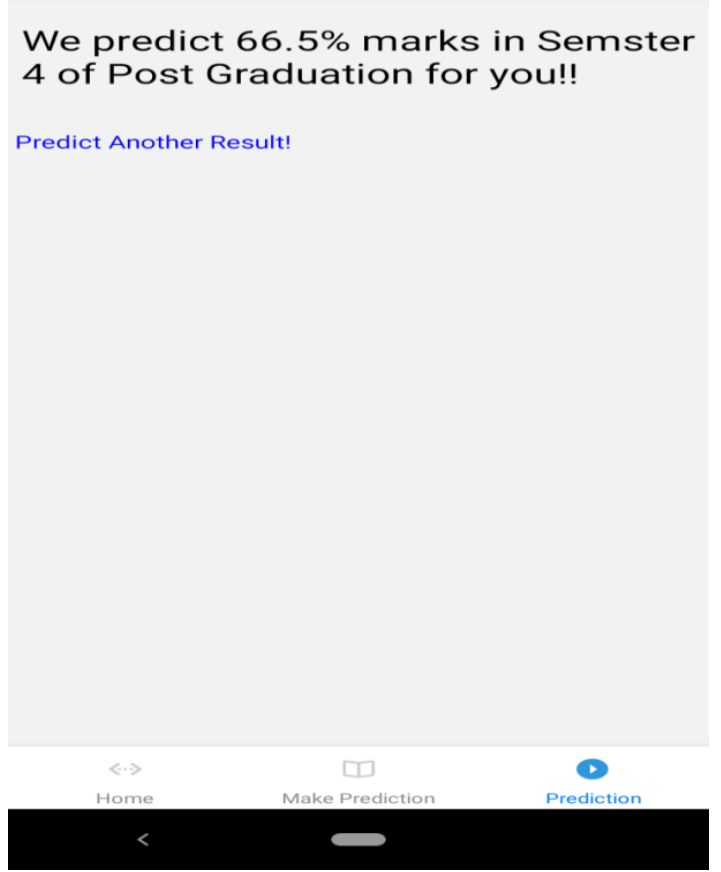

Figure 10. Predicted Screen for Student Result 


\section{MAINTENANCE}

Maintenance is the last phase, and it is a continuous process. Students can fill up feedback form to improve the developed App. Feedbacks can be collected and analyzed for the improvements. The APPA application can help students track the results of the last semester prior to the exam. Hence MADLC is successfully implemented in the Academic Performance Prediction Application (APPA) development process. This model helps researcher create an application efficiently.

\section{SCOPE FOR APPA IMPROVEMENTS}

- Implementing with other factors influencing student academic performance

- Extending the capabilities of the system

- Integrating Academic real time Dashboards

- Making it generalize amongst different courses

- Collaboration with University and Parent

- The university can publish content as guidelines for students which may be scared about their study.

- The app can also give guidelines based upon different personal and academic details they provide in order to score well in the future.

\section{CONCLUSION}

The performance of students is a crucial prerequisite to help students that doesn't performs well in the examination and can impact the final semester result. To overcome the difficulties they come across while learning and assist them to achieve best results. Researcher uses the advantages of the React-Native platform to build an "APPA" mobile application capable of delivering student performance prediction-related solution. The created app executes on android and $\mathrm{iOS}$, and the results produced represent satisfactory user experience in both platforms. Students will take actions to progress the result which will display and thereafter improve student 
performance. Faculty should also inspire students to do better throughout the semester and to direct them accordingly. APPA acquires the intention to design and develop predictive model and deploy the developed training to enhance student performance.

\section{REFERENCES}

[1] Fenty, E. K. (2014). Applying Mobile Application Development Life Cycle in the Development of Zakat Maal Mobile Web Application Using JQuery Mobile Framework . International Conference on Cyber and IT Service Management.

[2] KARTHIK_P. (2016). Bicycle model a new SDLC mode for an individual mobile application developer. NATIONAL CONFERENCE ON HUMAN COMPUTER INTERACTION (pp. 63-68). Tamil Nadu: International Journal of Advanced Research in Basic Engineering Sciences and Technology.

[3] Muhammad Adil, A. \&. (2015). Color Card Game (Project Report). Pakistan: Comsats Institute Of Information Technology.

[4] Nurfarah Ain, A. Z. (2015). Fast Track: Fasting Replacement Recommender Mobile Application Using Rule Based. . Malaysia: Universiti Teknologi MARA.

[5] Sawant, T. U., \& Pol, U. R. (2021). A Case Study on Machine Learning to Predict the Students' Result in Higher Education. In S. J. Vishal Jain, Handbook of Machine Learning for Computational Optimization (p. 294). Boca Raton: CRC Press.

[6] Sommerville, I. (2016). Software Engineering, 10th Edition. United Kingdom: Pearson.

[7] Vithani, T., \& Kumar, A. (2014). Modeling the Mobile Application Development Lifecycle. Proceedings of the International MultiConference of Engineers and Computer Scientists . Hong Kong. 